\title{
Thermodynamics of a Fermi liquid beyond the low-energy limit
}

\author{
Andrey V. Chubukov ${ }^{1}$, Dmitrii L. Maslov ${ }^{2}$, Suhas Gangadharaiah ${ }^{2}$, and Leonid I. Glazman ${ }^{3}$ \\ ${ }^{1}$ Department of Physics, University of Maryland, College Park, MD 20742-4111 \\ ${ }^{2}$ Department of Physics, University of Florida, P. O. Box 118440, Gainesville, FL 32611-8440 \\ ${ }^{3}$ Theoretical Physics Institute, University of Minnesota, Minneapolis, MN 55455
}

(Dated: June 11, 2018)

\begin{abstract}
We consider the non-analytic temperature dependences of the specific heat coefficient, $C(T) / T$, and spin susceptibility, $\chi_{s}(T)$, of $2 \mathrm{D}$ interacting fermions beyond the weak-coupling limit. We demonstrate within the Luttinger-Ward formalism that the leading temperature dependences of $C(T) / T$ and $\chi_{s}(T)$ are linear in $T$, and are described by the Fermi liquid theory. We show that these temperature dependences are universally determined by the states near the Fermi level and, for a generic interaction, are expressed via the spin and charge components of the exact backscattering amplitude of quasi-particles. We compare our theory to recent experiments on monolayers of $\mathrm{He}^{3}$.
\end{abstract}

PACS numbers:

The Landau Fermi Liquid (FL) theory states that the low-energy properties of an interacting fermionic system are determined by the states in the vicinity of the Fermi surface, and are similar to that of weakly interacting quasi-particles. At the lowest temperatures, when decay of quasi-particles can be neglected, the specific heat $C(T) \propto T$ and spin susceptibility $\chi_{s}(T)=$ const of a FL differ from the corresponding quantities for the Fermi gas only via the renormalizations of the effective mass and $g-$ factor [1]. However, this low-temperature limit of the FL theory, considered by Landau, cannot tell whether the subleading terms in $T$ are analytic, and whether they come only from low-energy states (and are therefore described by the FL theory) or from the states far away from the Fermi surface.

For non-interacting fermions, the subleading terms in $C(T) / T$ and $\chi_{s}(T)$ scale as $T^{2}$ and come from highenergy states. However, it was found back in the 60 s that in $3 \mathrm{D}$ systems, the leading correction to $C(T) / T$ due to interaction with either phonons [2] or paramagnons [3] is non-analytic in $T\left(T^{2} \ln T\right)$ and comes from the states in the immediate vicinity of the Fermi surface. The same result was later shown to hold for the electron-electron interaction [4]. More recently, it was shown by various groups [5, 6, 7, 8, , 9, 10] that the temperature dependence of $C(T) / T$ is also non-analytic in $2 \mathrm{D}$ and starts with a linear-in- $T$ term. The same behavior was also found for the uniform spin susceptibility [6, 8, 9, 10]. Two of us have argued [ $[\underline{6}$ that, to second order in short-range interaction, these linear-in- $T$ terms originate exclusively from scattering of fermions with zero total momentum and either small or near $2 k_{F}$ momentum transfers ( "backscattering").

In this paper, we consider the specific heat and spin susceptibility for a generic 2D Fermi liquid. In this case 1], the leading (constant) terms in $C(T) / T$ and $\chi_{s}(T)$ are expressed via the two harmonics $-F_{c}^{(1)}$ and $F_{s}^{(0)}$ - of the quasiparticle interaction function $F(\theta)$, or, equivalently, via the same harmonics of the scattering amplitude $A(\theta)$ ( $c$ and $s$ refer to charge and spin components). We show that the linear-in- $T$ terms in $C(T) / T$ and $\chi_{s}(T)$ are also universally expressed via the scattering amplitude, but they are determined by $A(\theta)$ at a particular angle $\theta=\pi$, rather than by $A(\theta)$ averaged over the Fermi surface. As there is no simple relation between $A(\pi)$ and $F(\pi)$, these subleading terms cannot be simply expressed via the Landau function. The only exception is a weakly screened Coulomb interaction, when the $T$-term in $C(T) / T$ can be expressed via $F(\pi)$.

To shorten the presentation, we discuss in some detail the calculation for $C(T)$, and then just present the result for $\chi_{s}(T)$, which can obtained in a similar manner [11]. The most straightforward way to obtain $C(T)$ beyond the leading term in $T$ is to find the thermodynamic potential $\Xi(T)$ within the Luttinger-Ward approach [12], and use $C(T)=-T \partial^{2} \Xi / \partial^{2} T$. The non-analyticity of $C(T) / T$ obviously originates from the non-analyticity of $\Xi(T)$. The thermodynamic potential $\Xi$ is expressed as

$\Xi=\Xi_{0}-2 T \sum_{\omega_{m}} \int \frac{d^{2} k}{4 \pi^{2}}\left[\ln \left[G_{0} G^{-1}\right]-\Sigma G+\sum_{\nu} \frac{1}{2 \nu} \Sigma_{\nu} G\right]$,

where $\Xi_{0}$ is the thermodynamic potential of the free Fermi gas per unit area, $G_{0}=\left(i \omega_{m}-\epsilon_{k}\right)^{-1}, G=$ $\left(i \omega_{m}-\epsilon_{k}+\Sigma\right)^{-1}, \Sigma$ is the exact (to all orders in the interaction) self-energy, and $\Sigma_{\nu}$ is the skeleton selfenergy of order $\nu$. Both $\Sigma_{\nu}$ and the sum $\Sigma=$ $\sum_{\nu} \Sigma_{\nu}$ are evaluated at finite $T$. Diagrams associated with the first two terms in (11) correspond to the selfenergy insertions into the free thermodynamic potential $\Xi_{0}=2 T \sum_{\omega_{m}} \int d^{2} k /(2 \pi)^{2} \ln G_{0}\left(\omega_{m}, k\right)$, diagrammatically represented by a loop (Fig. 11a). One can readily verify that such diagrams simply renormalize the constant term in $C(T) / T$. The non-analytic temperature dependence of $C(T) / T$ comes from the third term in (1).

To understand the origin of the non-analyticity in $\Xi(T)$, consider first a weak short-range interaction $U(q)$. To second order in $U$, the skeleton term gives rise to di- 


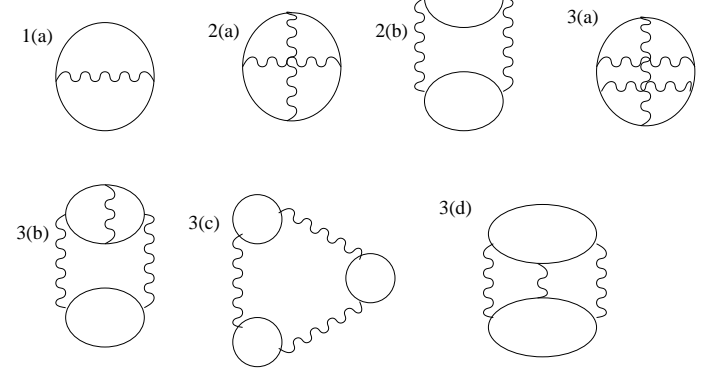

FIG. 1: Non-trivial second-order and third-order Diagrams for the thermodynamic potential. For the Coulomb potential, diagrams 1(a), 2(b) and 3(c) represent ring series.

agrams $2 \mathrm{a}$ and $2 \mathrm{~b}$ in Fig. 1 Assume momentarily that $U$ is a constant. Then each of the two diagrams can be re-expressed as a product of two particle-hole bubbles $\Pi\left(q, \Omega_{m}\right)$, so that

$$
\delta \Xi=-\frac{1}{2} T U^{2} \sum_{\Omega} \int \frac{d^{2} q}{4 \pi^{2}} \Pi^{2}\left(q, \Omega_{m}\right)
$$

where $\delta \Xi \equiv \Xi-\Xi_{0}$.

It is intuitively clear that the non-analyticity in $\delta \Xi$ should be related to a non-analyticity in $\Pi\left(q, \Omega_{m}\right)$. There are two regions of $q$ where $\Pi$ is non-analytic. First region is near $q=0$, where $\Pi\left(q, \Omega_{m}\right)=$ $-(m / 2 \pi)\left(1-\left|\Omega_{m}\right| /\left(\Omega_{m}^{2}+\left(v_{F} q\right)^{2}\right)^{1 / 2}\right)$. For $v_{F} q \gg$ $\left|\Omega_{m}\right|$, the Landau-damping term $\left(\left|\Omega_{m}\right| / q\right)$ is non-analytic in q. This non-analyticity leads to a long-range tail of $\Pi\left(r, \Omega_{m}\right)$ in real space: $\Pi\left(r, \Omega_{m}\right) \propto\left|\Omega_{m}\right| / r$. The second region is near $2 k_{F}$, where $\Pi\left(q, \Omega_{m}\right)=$ $-(m / 2 \pi)\left(1-\left(\bar{q}+\sqrt{\bar{q}^{2}+\bar{\Omega}_{m}^{2}}\right)^{1 / 2}\right)$, with $\bar{q}=(q-$ $\left.2 k_{F}\right) / 2 k_{F}$ and $\bar{\Omega}=\Omega / 2 k_{F} v_{F}$. The singularity at $\bar{q}=0$ is known as the Kohn anomaly. Most of the effects caused by the Kohn anomaly - Friedel oscillations, KohnLuttinger pairing - are associated with the (asymmetric) singularity in the static bubble: $\Pi\left(\bar{q}, \Omega_{m}=0\right)-$ $\Pi(0,0) \propto \theta(\bar{q}) \sqrt{q}$. However, the static Kohn anomaly does not cause a non-analytic $T$-dependence of $\delta \Xi(T)$ in (2) as the momentum integral over static $\Pi(q, 0)$ is infrared convergent. The non-analyticity in $\delta \Xi(T)$ comes from the dynamic Kohn anomaly, which is a term $|\bar{\Omega}| / \sqrt{|\bar{q}|}$ in $\Pi\left(\bar{q}, \Omega_{m}=0\right)$ for $-\bar{q} \gg\left|\Omega_{m}\right| / v_{F}$. This term leads to a dynamic Friedel oscillation: $\Pi\left(r, \Omega_{m}\right) \propto$ $\left|\Omega_{m}\right| \cos \left(2 k_{F} r\right) / \sqrt{r}$.

Integrating in Eq. (2) over the two momentum regions where $\Pi\left(q, \Omega_{m}\right)$ is non-analytic, we find that each of these two regions contributes a logarithmic singularity of the form $\Omega_{m}^{2} \ln \left|\Omega_{m}\right|$, the prefactors are the same. This logarithmic singularity is the key effect. Had it been absent, the Matsubara sum of $\Omega_{m}^{2}+\Omega_{m}^{4}+\ldots$ would have been controlled by high frequencies, of order $E_{F}$, and would have led to an analytic expansion $\delta \Xi=$ const $+T^{2}+T^{4}+\ldots$. The presence of the logarithm changes the story, as now the frequency sum contains a universal contribution from frequencies of order $T$. Using the Euler-Maclaurin summation formula, we obtain

$$
\begin{gathered}
\delta \Xi=-\frac{4 \pi u^{2} T^{3}}{v_{F}^{2}} S(M), \quad S(M) \equiv \sum_{m=0}^{M} m^{2} \ln \frac{M}{m} \\
=\frac{1}{9} M^{3}-\frac{1}{12} M-\frac{\zeta(3)}{4 \pi^{2}}+\frac{1}{360 M}+\ldots
\end{gathered}
$$

where $u=m U / 2 \pi$, and $M \sim E_{F} / 2 \pi T \gg 1$. Those terms in (3) that depend on $M$ yield a regular expansion for $\delta \Xi$ in powers of $T^{2}$, whereas the $M$-independent term - the third term in the second line of Eq. (3) - gives rise to $\delta \Xi \propto T^{3}$, and hence to a $T^{2}-$ term in $C(T)$.

Next, we take a more careful look at which fourfermion vertices actually contribute to the non-analytic part of $C(T)$. For the $2 k_{F}$-part, the answer follows immediately from the observation that, for a given direction of $\mathbf{q}$, the Kohn anomaly (both static and dynamic) comes from the internal fermionic momenta near $\mathbf{q} / 2$ and $-\mathbf{q} / 2$ in both bubbles in diagrams $2 \mathrm{a}$ and $2 \mathrm{~b}$ of Fig 1 The relevant vertex then has the momentum structure $(\mathbf{k},-\mathbf{k} ;-\mathbf{k}, \mathbf{k})$, which corresponds to backscattering.

For the $q=0$ part, the momentum structure is less obvious as, at the first glance, the internal momenta in the two bubbles in diagrams $2 \mathrm{a}$ and $2 \mathrm{~b}$ are uncorrelated. However, the logarithmic singularity of the momentum integral comes only from the $\left|\Omega_{m}\right| / q-$ part of each polarization bubble. One can show that this part comes from the integration over that special region where the internal momenta are nearly orthogonal to $\mathbf{q}$. Since the relevant momenta in the two bubbles are almost orthogonal to the same vector (q), they must be either nearly parallel or nearly antiparallel to each other. We verified that the contribution from the near parallel momenta, i.e., from forward scattering, vanishes and the full result comes from nearly antiparallel momenta. This implies that the $q=0$ contribution to $\delta \Xi$ involves a vertex with the momentum structure $(\mathbf{k},-\mathbf{k} ; \mathbf{k},-\mathbf{k})$. This vertex is also a part of the backscattering amplitude.

We can now extend our second-order analysis to a finite-range interaction $U(q)$. That only backscattering is relevant means that only $U(0)$ and $U\left(2 k_{F}\right)$ contribute to the $T$-term in $C(T) / T$. The contribution of the diagram $2 \mathrm{~b}$ is proportional to $U^{2}(0)+U^{2}\left(2 k_{F}\right)$, whereas that of the diagram $2 \mathrm{a}$ is proportional to $U(0) U\left(2 k_{F}\right)$. Collecting the prefactors, we obtain for the non-analytic part of the specific heat $\Delta C \equiv C(T)-\gamma T$

$$
\Delta C(T) / T=-\left(u_{0}^{2}+u_{2 k_{F}}^{2}-u_{0} u_{2 k_{F}}\right) \frac{3 m \zeta(3)}{\pi} \frac{T}{E_{F}}
$$

where $u_{0}=m U(0) / 2 \pi$ and $u_{2 k_{F}}=m U\left(2 k_{F}\right) / 2 \pi$. This 
agrees with the result obtained in Ref. [6] by expressing $C(T) / T$ via the self-energy.

Consider now what happens when we add higher-order terms in $U$. They lead to two types of corrections: selfenergy corrections to the fermionic lines in the two bubbles and corrections to the four-fermion vertices. The self-energy corrections are of the FL type: they account for the appearance of the quasiparticle $Z$-factors and for the replacement of the bare fermionic mass $m$ by $m^{*}$. Vertex corrections generate terms with more bubbles. A generic diagram of $n$-th order has $n$ bubbles. To obtain a $T^{3}$-contribution to $\Xi(T)$, we need to take dynamic, $\Omega_{m} / Q$-terms from two bubbles out of $n$ and set $\Omega_{m}=0, Q \rightarrow 0$ in the rest $n-2$ bubbles, because any extra power of $\Omega_{m} / Q$ eliminates the logarithmic singularity in the frequency integrand in $\delta \Xi(T)$. It is intuitively plausible that once two dynamic bubbles are chosen at the $n$-th order, the rest of the $n$-th order diagram constitutes the $n$-th order correction to the static four-point vertex. If this conjecture is true, the series of the diagrams for the non-analytic $T^{3}-$ term in the thermodynamic potential can be re-expressed in terms of the two-bubble diagrams in which $U(0)$ and $U\left(2 k_{F}\right)$ are replaced by exact static vertices $\Gamma(\mathbf{k},-\mathbf{k} ; \mathbf{k},-\mathbf{k})$ and $\Gamma(\mathbf{k},-\mathbf{k} ;-\mathbf{k}, \mathbf{k})$. Accordingly, $\Delta C(T) / T$ is given by the same expression as in (4), but with $\Gamma$ instead of $U$.

This conjecture, however, needs to be verified as different diagrams for the thermodynamic potentials contain different combinatorial factors, and it is a priori unclear whether these factors, combined with those counting the number of ways two dynamic bubbles can be chosen, give the right coefficients in the perturbative series for the static vertices. To verify that this is the case, we evaluated explicitly the $T^{3}$-term in the thermodynamic potential to third order in $U(q)$, and compared the result with that given by the two-bubble diagrams with the renormalized static vertices, evaluated independently. We found that the two expressions are identical. In what follows, we assume that this equivalence survives to all orders in $U(q)$.

The renormalization from $U(0)$ and $U\left(2 k_{F}\right)$ to $\Gamma(\mathbf{k},-\mathbf{k} ; \mathbf{k},-\mathbf{k})$ and $\Gamma(\mathbf{k},-\mathbf{k} ;-\mathbf{k}, \mathbf{k})$ includes static corrections coming from the states both away and near the Fermi surface (the latter produce powers of static $\left.\Pi\left(\Omega_{m}=0, Q \rightarrow 0\right)=-m / 2 \pi[1]\right)$. In other words, $\Gamma(\mathbf{k},-\mathbf{k} ; \mathbf{k},-\mathbf{k})$ and $\Gamma(\mathbf{k},-\mathbf{k} ;-\mathbf{k}, \mathbf{k})$ include all vertex corrections except for the terms coming from the dynamic part of the polarization bubble. In conventional notations [1], the fully renormalized $\Gamma(\mathbf{k},-\mathbf{k} ; \mathbf{k},-\mathbf{k})$ and $\Gamma(\mathbf{k},-\mathbf{k} ;-\mathbf{k}, \mathbf{k})$ are then related to $\Gamma^{k}(\theta=\pi)$, which is the limit of $\Omega=0$ and $q \rightarrow 0$ of $\Gamma(\mathbf{k}, \mathbf{p}, \mathbf{k}+\mathbf{q}, \mathbf{p}-\mathbf{q})$, where both $\mathbf{k}$ and $\mathbf{p}$ are on the Fermi surface, and $\theta$ is the angle between these two vectors.

The matrix (in the spin space) $\hat{\Gamma}^{k}(\pi)$ is related to the quasiparticle scattering amplitude via $\hat{A}(\pi)=Z^{2} \hat{\Gamma}^{k}(\pi)$ [1]. Decomposing the scattering amplitude for a spin- invariant interaction into charge and spin components $A_{c}$ and $A_{s}$ as

$$
\hat{A}(\pi)=\frac{\pi v_{F}^{*}}{k_{F}}\left[A_{c}(\pi) \hat{I}+A_{s}(\pi) \hat{\sigma} \cdot \hat{\sigma}\right]
$$

and comparing (5) with a similar decomposition for $\hat{\Gamma}^{k}(\pi)$

$$
\begin{aligned}
\hat{\Gamma}^{k}(\pi)= & \Gamma^{k}(\mathbf{k},-\mathbf{k} ; \mathbf{k},-\mathbf{k}) \hat{I} \\
& -(1 / 2) \Gamma^{k}(\mathbf{k},-\mathbf{k} ;-\mathbf{k}, \mathbf{k})(\hat{I}+\hat{\sigma} \cdot \hat{\sigma}),
\end{aligned}
$$

we obtain

$$
\begin{aligned}
Z^{2} \Gamma^{k}(\mathbf{k},-\mathbf{k}, \mathbf{k},-\mathbf{k}) & =\frac{\pi v_{F}^{*}}{k_{F}}\left[A_{c}(\pi)-A_{s}(\pi)\right] \\
Z^{2} \Gamma^{k}(\mathbf{k},-\mathbf{k} ;-\mathbf{k}, \mathbf{k}) & =-2 \frac{\pi v_{F}^{*}}{k_{F}} A_{s}(\pi)
\end{aligned}
$$

Substituting $Z^{2} \Gamma^{k}(\mathbf{k},-\mathbf{k}, \mathbf{k},-\mathbf{k})$ instead of $U(0)$ and $Z^{2} \Gamma^{k}(\mathbf{k},-\mathbf{k} ;-\mathbf{k}, \mathbf{k})$ instead of $U\left(2 k_{F}\right)$ into (4), we find the non-analytic part of $C(T)$ in a generic Fermi liquid

$$
\Delta C(T) / T=-\left(\frac{3 \zeta(3)}{2 \pi}\right)\left(\frac{m^{*}}{k_{F}}\right)^{2} \quad\left[A_{c}^{2}(\pi)+3 A_{s}^{2}(\pi)\right] T .
$$

A similar generalization of the second-order result for $\chi_{s}(T)[6]$ yields

$$
\Delta \chi_{s}(T)=\frac{m^{*}}{4 k_{F}^{2}} \chi_{s}(0) A_{s}^{2}(\pi) T
$$

where $\chi_{s}(0)$ is the spin susceptibility at $T=0$.

Eqs. (8) and (9) are the two main results of the paper. We see that the non-analytic parts in $C(T)$ and $\chi_{s}(T)$ are parameterized by two scattering amplitudes, $A_{c}(\pi)$ and $A_{s}(\pi)$. These amplitudes are the new parameters in an extended version of the FL theory, which includes non-analytic terms. Notice that $A_{c}(\pi)$ and $A_{s}(\pi)$ cannot be simply expressed in terms of the quasiparticle interaction function $F(\theta)$, which, we remind, is related to $Z^{2} \hat{\Gamma}^{\omega}(\theta)=\left(\pi v_{F}^{*} / k_{F}\right)\left(F_{c}(\theta) \hat{I}+F_{s}(\theta) \hat{\sigma} \cdot \hat{\sigma}\right)$. The harmonics $A_{a}^{(n)}$ and $F_{a}^{(n)}(a=c, s)$ are indeed simply related, $A_{a}^{(n)}=F_{a}^{(n)} /\left(1+F_{a}^{(n)}\right)$ in $2 \mathrm{D}$, but $A_{a}(\pi)$ is only expressed via infinite series of harmonics of the Landau function:

$$
A_{a}(\pi)=\sum_{n=0}^{\infty}(-1)^{n} F_{a}^{(n)} /\left(1+F_{a}^{(n)}\right)
$$

Rather involved explicit relations between $A_{a}(\pi)$ and $F_{a}(\pi)$ to third order in $U$ are presented in [13].

We find, however, that a simple relation between $\hat{A}(\pi)$ and $\hat{F}(\pi)$ does exist if the interaction $U(q)$ is strongly peaked at $q=0$. In this situation, only corrections due to $U(0)$ matter. These corrections come from ring diagrams and can be summed up exactly [the combinatorial factor for a ring diagram of order $n$ is $\left.(-1)^{n} 2^{n-2}(n-1)\right]$. 
Evaluating the sum over $n$, we obtain $A_{c}(\pi)=F_{c}(\pi) /(1+$ $\left.F_{c}(\pi)\right)$. If, in addition, $F_{s}(\theta) \ll 1$, the contribution to the specific heat from $F_{c}(\pi)$ dominates, and the singular term in the specific heat becomes

$$
\Delta C(T) / T=-\frac{3 m \zeta(3)}{4 \pi} \frac{T}{E_{F}}\left(\frac{F_{c}(\pi)}{1+F_{c}(\pi)}\right)^{2} .
$$

If $F_{c}(\pi)$ is dominated by the $n=0$ harmonic, Eq. (11) coincides with the result of Ref. 14.

The limit $F_{c}(\pi) \rightarrow \infty, F_{s}(\theta) \ll 1$ corresponds to the Coulomb interaction for small $r_{s}$. In this limit, $F_{c}(\pi)$ is canceled out from (11), and the singular term in the specific heat becomes

$$
\Delta C(T) / T=-\frac{3 m \zeta(3)}{4 \pi} \frac{T}{E_{F}} .
$$

In agreement with Refs. 7, 14], we find that for the Coulomb interaction, the $T^{2}$ term in the specific heat is independent of $r_{s}$ for small $r_{s}$.

Scattering amplitudes in Eqs. (8) and (9) can be extracted from a measurement of $C(T) / T$ and $\chi_{s}(T)$ on the same system. To the best of our knowledge, a linearin- $T$ dependence of $\chi_{s}$ has not been measured yet. However, the linear temperature dependence of $C(T) / T$ has been observed in several experiments on fluid monolayers $\mathrm{He}^{3}$ adsorbed on graphite [15, 16, 17]. To a reasonable accuracy, the data can be fitted into a form $C /\left(N T / E_{F}^{*}\right)=\gamma\left(T / E_{F}^{*}\right)$, where $N$ is the density per unit area in a fluid monolayer, $E_{F}^{*}=E_{F}\left(\mathrm{~m} / \mathrm{m}^{*}\right)$ and $\gamma(x) \approx a-b x$ for small $x$ [17]. Both $a$ and $b$ vary somewhat with $N$, but the variation is not dramatic, and to reasonable accuracy $a \sim 3-3.3$, and $b \sim 10-14$ 19]. According to Eqs. (33) and (8), $a=\pi^{2} / 3 \approx 3.3$, and $b=0.9\left[A_{c}^{2}(\pi)+3 A_{s}^{2}(\pi)\right]$. A fit to the data then yields $A_{c}^{2}(\pi)+3 A_{s}^{2}(\pi) \approx 11-15.5$ [19]. To estimate $A_{c}(\pi)$ and $A_{s}(\pi)$ separately, we assume that the scenario of "almost localized fermions" 18], which describes successfully the properties of bulk $\mathrm{He}^{3}$, is applicable to the $2 \mathrm{D}$ case as well. In this scenario, the interaction in the charge channel is strong, whereas that in the spin channel is moderate. A strong interaction in the charge channel means that $F_{c}^{(n)} \gg 1$, in which case the consecutive terms in series for $A_{c}(\pi)$ [Eq. (10)] almost cancel each other, and the result is likely to be small. A precsie value for $A_{c}(\pi)$ depends on how $F_{c}^{(n)}$ decrease with $n$. However, in two model cases $F_{c}^{(n)}=g /\left(1+n^{2}\right)$ and $F_{c}^{(n)}=g e^{-n}$, we obtain almost identical results: $A_{c}^{2}(\pi) \approx 0.25$ in the limit of $g \gg 1$. This suggests that the observed value $A_{c}^{2}(\pi)+3 A_{s}^{2}(\pi) \approx 11-15.5$ is almost entirely due to the spin part of the amplitude. Neglecting $A_{c}^{2}(\pi)$, we obtain $\left|A_{s}(\pi)\right| \approx 1.9-2.3$. If the $n=0$ harmonic of $F_{s}$ dominates the result for $A_{s}(\pi)$, i.e., $A_{s}(\pi) \approx F_{s}^{(0)} /\left(1+F_{s}^{(0)}\right)$, then $F_{s}^{(0)} \approx-(0.66-0.7)$, which is consistent with the $3 \mathrm{D}$ value $F_{s}^{(0)} \approx-0.75[20]$.
Notice also that if $A_{c}(\pi)$ can be neglected compared to $A_{s}(\pi), \Delta C(T) / T$ and $\delta \chi_{s}(T)$ contain only one unknown parameter $\left(A_{s}(\pi)\right)$. In this situation, the ratio $K=\Delta C(T) /\left(T \Delta \chi_{s}(T)\right)$ is expressed only via the parameters describing the leading, analytic parts of $C(T)$ and $\chi_{s}: K=-18 \zeta(3) m^{*} / \pi_{s}(0)$.

In addition, $A_{s}(\pi)$ determines the slope of the linearin- $T$ correction $\Delta \sigma(T)$ to the conductivity of a dirty 2D FL in the ballistic regime 21], which allows to express $\delta \chi_{s}(T)$ in terms of $\Delta \sigma(T)$ as $\Delta \chi_{s}(T) E_{F}^{*} / \chi_{s}(0) T=$ $\left[1-\Delta \sigma(T) E_{F}^{*} / \sigma(0) T\right]^{2} / 72$. These predictions are amenable to a direct experimental verification.

To summarize, in this paper we showed that the $2 \mathrm{D}$ Fermi-liquid theory describes not only the leading, constant terms in the specific heat coefficient $C(T) / T$ and the spin susceptibility $\chi_{s}(T)$, but also subleading, linearin- $T$ terms. We argued that these terms come from backscattering, and are universally expressed via the spin and charge components of the scattering amplitude at the angle $\theta=\pi$. We extracted the spin component of the scattering amplitude from the experimental data on $C(T)$ for a monolayer of $\mathrm{He}^{3}$.

We acknowledge stimulating discussions with I. Aleiner, B. Altshuler, A. Andreev, A. Millis, and J. Saunders. The research has been supported by NSF Grant No. DMR 0240238 (A. V. Ch.), NSF Grant No. DMR0308377 (D. L. M.), and NSF Grant No. DMR-0237296 (L. I. G.).

[1] A. A. Abrikosov, L. P. Gorkov, and I. E. Dzyaloshinski, Methods of quantum field theory in statistical physics, (Dover Publications, New York, 1963); E. M. Lifshitz and L. P. Pitaevski, Statistical Physics, (Pergamon Press, 1980).

[2] G. M. Eliashberg, Sov. Phys. JETP 16, 780 (1963).

[3] S. Doniach and S. Engelsberg, Phys. Rev. Lett. 17, 750 (1966); W. F. Brinkman and S. Engelsberg, Phys. Rev. 169417 (1968).

[4] D. J. Amit, J. W. Kane, and H. Wagner, Phys. Rev. 175, 313 (1968); ibid. 175, 326 (1968).

[5] D. Coffey and K. S. Bedell, Phys. Rev. Lett. 71, 1043 (1993).

[6] A. V. Chubukov and D. L. Maslov, Phys. Rev. B 68, 155113 (2003); ibid. 69, 121102 (2004).

[7] V. M. Galitski and S. Das Sarma, Phys. Rev. B 70, 035111 (2004).

[8] D. Belitz, T. R. Kirkpatrick, and T. Vojta, Phys. Re v. B 55, 9452 (1997)

[9] M. A. Baranov, M. Yu. Kagan, and M. S. Mar'enko, JETP Lett. 58, 709 (1993).

[10] G. Y. Chitov and A. J. Millis, Phys. Rev. Lett. 86, 5337 (2001); ibid. Phys. Rev. B 64, 0544414 (2001).

[11] V. M. Galitski, A. V. Chubukov, and S. DasSarma, cond-mat/0501132 J. Betouras, D. Efremov, and A. Chubukov, unpublished.

[12] J. M. Luttinger and J. C. Ward, Phys. Rev. 118, 1417 (1960). 
[13] A. V. Chubukov, D. L. Maslov, S. Gangadharaiah, and L. I. Glazman, cond-mat/0412283

[14] G. Catelani and I. L. Aleiner, cond-mat/04053333.

[15] D.S. Greywall, Phys. Rev. B 41, 1942 (1990).

[16] M. Ocura and H. Hamaizawa, J. Phys. Soc. Jpn, 66, 3706 (1997).

[17] A. Casey, H. Patel, J. Nyeki, B.P. Cowan and J. Saunders, Pys. Rev. Lett. 90, 115301 (2003).

[18] D. Vollhardt, Rev. Mod. Phys., 56, 99 (1984).

[19] This value of $B$ is also consistent with earlier measure- ments by Greywall 15] and Wada et al 16], once one eliminates the rapid rise of $C / T$ at low $T$. This rapid rise is most likely an artifact caused by of the substrate heterogeneity. Ocura and Hamaizawa [16] used the lowest $T$ data to fit the slope of $C(T) / T \propto T$. Our results are inconsistent with theirs.

[20] D.S. Greywall, Phys. Rev. B 27, 2747 (1983).

[21] G. Zala, B. N. Narozhny, I. L. Aleiner, Phys. Rev. B 64, 214204 (2001). 